\title{
SURGIMIENTO Y RUPTURA DE LA SOCIOLOGÍA ALEMANA: ENTRE EL IMPERIO, LA REPÚBLICA Y EL RÉGIMEN NAZI
}

\author{
RISE AND RUPTURE OF THE GERMAN SOCIOLOGY: BETWEEN THE \\ EMPIRE, THE REPUBLIC AND THE NAZI REGIME
}

\author{
Hellmut Wollmann \\ Universidad Humboldt, Berlín. Alemania/Germany \\ hellmut.wollmann@,rz.hu-berlin.de
}

Recibido/Received: 18/11/2013

Modificado/Modified: 30/01/2014

Aceptado/Accepted: 20/06/2014

\section{RESUMEN}

El artículo describe la emergencia y ruptura de la sociología alemana desde sus inicios a principios del siglo XX hasta 1933 en la Universidad en Berlín, fundada en 1810 y desde entonces centro del mundo universitario alemán. Durante su fase inicial, fue modelada por Gustav Schmoller, un típico economista de corte reformista (de la "Escuela Histórica"). El despliegue disciplinario posterior de la sociología fue promovido por una cohorte de graduados, entre los cuales se destacaron Max Weber, Georg Simmel y Werner Sombart. Tras su florecimiento durante la década del veinte, fue "brutalmente interrumpido" (René König) cuando los nazis tomaron el poder, empujando a casi todos estos sociólogos al exilio o a la "emigración interna". Desde 1933, una nueva cohorte ocupó la escena universitaria, suministrando, por medio del instituto universitario de Berlín y de su vinculación con la SS, la base ideológica y "científica" de las políticas expansionistas y genocidas.

\section{PALABRAS CLAVE}

Universidad de Berlín, historia de los intelectuales, desarrollo del conocimiento sociológico.

\section{SUMARIO}

1. Antecedentes. 2. El desarrollo de la sociología entre 1918 y 1933. 3. La sociología bajo la dominación nazi. 4. Reflexiones. Bibliografía.

\begin{abstract}
The article pursues the emergence and rupture of German sociology between its beginning in the 19th century and 1933. It focuses on the University in Berlin which, founded in 1810, became the hub in Germany's university world. The early phase was significantly shaped by Gustav Schmoller who was typically a reformist-minded economist (of the "Historical School"). In its further disciplinary unfolding sociology was promoted by a cohort of scholars among whom Max Weber, Georg Simmel and Werner Sombart excelled. After flourishing during the 1920s it was "brutally terminated" (René König) in 1933 when the Nazis seized power driving almost the entire generation of sociologists into exile or "inner emigration". Since 1933 a new cohort of social scientists occupied the university scene that was ready to serve the Nazi rulers, with an institute at Berlin University, linked to the SS leadership, playing a particularly pernicious role.
\end{abstract}

\section{KEYWORDS}

University of Berlin, intellectuals' history, development of sociological knowledge. 


\section{CONTENTS}

1. Background. 2. The development of sociology between 1918 and 1933. 3. Sociology under the Nazi domain. 4. Conclusions. References.

\section{ANTECEDENTES}

El comienzo de la investigación y docencia en las ciencias sociales suele ser atribuido a la cameralística del siglo dieciocho (Wagner, 1990:85; Wagner y Wollmann, 1991). (El término alemán, Kameralistik, proviene del latín y alude a las arcas y finanzas del príncipe que se guardaban en las recámaras) A las "ciencias económicas, policey y camerales", que se desplegaron en el (tardo) Estado absolutista alemán del siglo XVII y XVIII, como doctrinas generales administrativas y de la "ciencia política del Estado territorial" (Maier, 1990:261), se les asignó la particular tarea de proveer información y datos empíricos útiles a la política económica mercantil de los señores territoriales y capacitar a su personal administrativo. Dentro de las ciencias camerales, ganó la estadística, cuya raíz lingüística hace referencia al "conocimiento del Estado (= status, stato, état)", de prominente importancia. Ejemplo de su institucionalización académica durante el reinado de Federico Guillermo I de Prusia en 1727, fue el establecimiento de cátedras de cameralística en las Universidades de Halle y Frankfurt/Oder. A principios de la economía capitalista del siglo XIX, con la transición de un Estado tardo-absolutista a otro de concepción liberal y de una economía mercantil-estatal a otra liberal-burguesa, las "ciencias policey y camerales" se diseñaron en enseñanza e investigación. A las ciencias del Estado preexistentes, incluida la estadística, las mantuvo el peculiar interés por el conocimiento empírico y la capacitación de orientación práctica. A su vez, la doctrina económica mercantil fue reemplazada progresivamente por una economía política de origen anglosajón.

Desde la fundación de la Universidad de Berlín en 1810, la ciencia del Estado y la estadística pertenecieron al campo de la enseñanza e investigación desplegado en la en la Facultad de Filosofía (Zschaler, 1996/97). Conforme a las sujeciones tradicionales de la enseñanza académica y la práctica administrativa, el primer titular de la cátedra de ciencias del Estado se desempeñó, al igual que su sucesor, en unión personal con la dirección de la Oficina Estadística Prusiana. Finalmente, en 1833 la economía política fue establecida como materia disciplinar en la Universidad de Berlín, entonces llamada Friedrich-WilhelmsUniversität. Luego de 1870, la enseñanza e investigación de las ciencias del Estado cobró un nuevo impulso, ya que en 1870 fueron llamados a Berlín el economista y financista, Adolf Wagner, y, en 1882, el economista e historiador económico, Gustav Schmoller. Entre ellos, estaban reunidos en la Universidad Federico Guillermo los dos economistas y "eruditos políticos gubernamentales" (Bruch, 1980; Käsler, 1984:269) más renombrados de la época de Bismarck. A título de una notable institucionalización de la investigación científico-estatal, fundaron en 1886 en colaboración con el historiador agrario y estadista, August Meitzen, y el demógrafo y estadista, Richard Boeckh, el Seminario Estadístico de Ciencias del Estado dentro de la Facultad de Filosofía.

Tanto Gustav Schmoller como Adolf Wagner -al igual que otros representantes de la Escuela Histórica-, vincularon a sus investigaciones una agenda, mediante las indagaciones sobre la "cuestión social", para señalar la necesidad y posibilidad de reformas estatales, lo que les valió, a ellos y a las personas con los mismos intereses, la irónica rotulación de "socialistas de cátedra". En 1872, ambos formaron parte, en igual medida, de la fundación de 
la Asociación de Política Social (Verein für Socialpolitik) en la que se congregaron profesores y funcionarios político-reformistas motivados por el objetivo de iniciar y emprender indagaciones empíricas (Enquêten) sobre la "cuestión social" (sobre la carencia de vivienda de la clase trabajadora) y revelar con ello la necesidad de una reforma estatal (Gorges, 1986:37; Wagner, 1990:80; Wagner y Wollmann, 1991:61). Desde 1890 hasta su muerte (1917), Schmoller presidió la Asociación. Las Enquêten, que fueron diseñadas y realizadas con un enorme esfuerzo metodológico y empírico, en parte -entre otros por Max Weber -, suministraron a partir de su ímpetu político-reformista considerables impulsos metódicos y conceptuales para el desarrollo de la investigación en ciencias sociales en Alemania. Hasta el cambio de siglo, la Asociación fue el hecho más importante de las ciencias sociales en Alemania y, en gran medida, un modelo considerable en el mundo occidental (Wagner, 1990:90). En esencia, Schmoller y Wagner contribuyeron, por su eficacia en la enseñanza, investigación y "política erudita" (vom Bruch), al carisma y atracción de la Universidad de aparecer, en un contexto nacional e internacional, como "meca" para los estudiantes locales y extranjeros y a modo de "destino final universitario, cumbre de la carrera académica" (Baumgarten, 1997:60).

Entre los estudiantes que buscaron la influencia académica de Schmoller y también de Wagner, formó parte una cohorte nacida alrededor de la década de 1860, en especial, Georg Simmel (nacido en 1858), Werner Sombart (1863), Max Weber (1864), Franz Oppenheimer (1864), Kurt Breysig (1866), Alfred Vierkandt (1867), Alfred Weber (1868), al igual que Leopold von Wiese (un tanto posterior, 1876). De estos, se doctoraron en Berlín: Simmel (1881), Sombart (1883), Oppenheimer (1885), Max Weber (1889), Breysig (1900), Alfred Weber (1900) (los dos Weber en la Facultad de Ciencias Jurídicas), von Wiese (1902). De estos, Bresysig, Simmel, Sombart, Oppenheimer, A. Weber y von Wiese, se consideraron explícitamente alumnos de Schmoller (Käsler, 1984:341). En la Universidad de Berlín obtuvieron su habilitación (facultas docendi, venia legendi en una disciplina científica): Simmel (1885), Vierkandt (1890), Max Weber (1892), Breysig (1892), Alfred Weber (1900), von Wiese (1905), (los dos Weber nuevamente de la Facultad de Ciencias Jurídicas). En vistas a la influencia que tuvo esta "cohorte de 1860" sobre el desarrollo de las ciencias sociales en Alemania, la Universidad se convirtió realmente durante esta fase en un "centro de elite" académico.

Si bien, Berlín contribuyó a la calificación, la Universidad, no obstante, no instauró la carrera académica, esto es, el empinado recorrido del Privatdozent hacia el de Profesor ordinario. A fines del siglo XIX, se llegó a lo habitual en el caso de las ciencias sociales, las restricciones se recrudecieron, sobre todo los filtros político-ideológicos en las políticas de nombramiento, de modo tal que un socialdemócrata, "socialista", o un crítico del Estado autoritario guillermino no tenía ninguna oportunidad de ser nombrado profesor ordinario, en esencia porque también el cuerpo de profesores era políticamente conservador, cuando no reaccionario o antisemita.

En el caso de Berlín, estas restricciones y filtros prevalecieron como fuertes barreras de acceso a la capital universitaria y fueron especialmente complicadas para la "cúspide" a una carrera académica, tal como se ilustra a continuación.

1) Georg Simmel se doctoró en 1881 (por Schmoller), enseñó filosofía tras su habilitación (1885), inicialmente (desde 1885) como Privatdozent y luego (desde 1900) como extraordinario ( $\sin$ un salario estable). Que se le haya negado a él una cátedra ordinaria, quien por treinta años enseñó en la Facultad de Filosofía, puede explicarse (Sigmund, 1993:175; Nedelmann, 1999:128-150, se contradice explícitamente con Gerhardt, Mehring y Rindert, 
2000:15) por los prejuicios antisemitas de sus colegas de la Facultad, tal como se hizo manifiesto en 1908 con su frustrado nombramiento para Heidelberg. (La designación de Simmel como profesor exclusivo fue denegada en 1908 a pesar de la manifiesta recomendación de Max Weber frente al contrario dictamen presentado por un colega historiador de la Universidad de Berlín (!), en el cual se dijo, entre otras cosas, que Simmel era "israelita de principio a fin, en su apariencia externa, su comportamiento y su mentalidad", citado en Nedelmann, 1999:128).

Por otro lado, la retención por treinta años a una titularidad también podría ser atribuida al hecho de haber quedado vinculado como representante de una sociología "filosófica", pero más que nada por haber quedado socialmente marginado del círculo de colegas. En 1914, obtuvo finalmente (a los 56 años) el nombramiento, como profesor ordinario de filosofía y pedagogía en la (más bien periférica) "Universidad Imperial" de Estrasburgo, en donde ejerció hasta su muerte (1918).

2) Franz Oppenheimer (1864-1943) (Oppenheimer, 1929:69-116; Eubank, 1934:60-178; Käsler, 1984:370) se doctoró en Medicina en Berlín en 1885 y en 1909 obtuvo su doctorado en Filosofía en la Universidad de Kiel, de 1909 hasta 1917 enseñó como Privatdozent en Berlín y desde 1917 hasta 1919 como profesor extraordinario. En tanto socialista (liberal), además por su descendencia judía, y más aún por ser un sionista (Bein) activo, difícilmente pudo contar con una cátedra ordinaria en la Prusia Guillermina. En 1919, fue convocado a una nueva cátedra ordinaria de Economía política y Sociología en el Instituto Superior creado por la Universidad de Frankfurt.

3) En el caso de Werner Sombart (1863-1941) (Käsler, 1984:422; Eubank, 1934:97; Lenger, 1994), quien se doctoró en 1888 (también por Schmoller), catalogado de "socialista" a razón de sus tempranos trabajos, los procedimientos para el nombramiento en Friburgo, Heidelberg y Karlsruhe fracasaron por objeción del Gran Duque de Baden, quien lo rechazó por radical de izquierda. En 1890, el "marxista" fue "neutralizado" (Stölting' 1986:112) con el nombramiento a una cátedra ordinaria en la (políticamente más periférica) Universidad de Breslau (de Prusia) y se mantuvo aquí durante 16 años. En 1906, obtuvo un nombramiento en el Instituto Superior de Comercio de Berlín, fundada como establecimiento privado por comerciantes berlineses en el mismo año y cuya política liberal de nombramiento lo aceptó como "izquierdista". Si bien, el ingreso al Instituto de Comercio abonó al deterioro de su reputación, estaba, sin embargo, finalmente en la "capital", cuya Universidad le había rechazado la posibilidad de dar clases. En los inicios de 1918, logró "superar la fuerte resistencia de la Universidad de Berlín y ser nombrado sucesor catedrático de Adolf Weber" (Käsler, 1984:426), inicialmente como extraordinario y luego, en 1919, como profesor ordinario de ciencias del Estado.

4) También, en el contexto de la Universidad Federico-Guillermo fue nombrado Hugo Preuß (1860-1925) (Faatz, 2002:15-27), quien, en 1889 había obtenido su habilitación en la Facultad de Derecho, a pesar de ser un "judío confeso" y activo políticamente de "izquierda", consiguió al mismo tiempo que Werner Sombart, antes de 1906, una cátedra en el Instituto Superior de Comercio de Berlín (del cual fue el rector en 1918). (En 1919 se convirtió en Ministro Imperial del Interior y realizó una significativa contribución en la elaboración de la constitución de Weimar).

5) Un ejemplo adicional sobre una carrera científica con fuerte limitaciones políticas ofrece el sociólogo, Ferdinand Tönnies (1855-1936). A raíz de sus tempranos trabajos fue clasificado bajo los ojos de los conservadores como "comunista", de esta forma perdió la posibilidad, tras su investigación sobre la huelga de los trabajadores portuarios de Kiel, de conseguir un nombramiento en la Universidad de Berlín (Käsler, 1984:403 y ss). Obtuvo su 
Habilitation (venia legendi) en 1881 en la Universidad de Kiel (Desde 1867 SchleswigHolstein fue una provincia prusiana) y enseñó durante treinta años como Privatdozent, antes de que el Ministerio de Cultura Prusiano lo nombrase profesor extraordinario en 1909 y a partir de 1913, finalmente (cuando tenía 69 años!), profesor ordinario (Bickel, 1999:113126). Su vida, como docente, la pasó en la Universidad periférica de Kiel, y su nombramiento para la "Universidad-terminal" de Berlín no fue nunca aceptado.

6) Por último, se debe hacer referencia al sociólogo, Robert Michelis (1875-1936) (Stölting, 1999:230-251), quien, a pesar de que Max Weber abogó públicamente por él, como "izquierdista" y miembro activo del Sozialdemokratische Partei Deutschlands en la Alemania Imperial no tuvo ninguna oportunidad en su carrera (y se fue a Italia, donde se unió al fascismo italiano).

Como se hizo visible en los ejemplos de Werner Sombart y Hugo Preuß, una apertura de pluralidad académica y liberalismo, en comparación con la práctica de nombramiento política e ideológicamente restringida, sobre todo para los judíos y los de "izquierda", los llevó del Instituto Superior de Comercio de Berlín, fundado en 1905, a la Universidad Federico-Guillermo. La disciplina apuntó así, entonces, a complementar el Seminario Estadístico Científico-estatal de orientación económico-popular, creado en 1886 (Zschaler, 1996).

Un paso importante para la institucionalización y consolidación de la sociología como disciplina autónoma fue la fundación en 1909 de la Sociedad Alemana de Sociología (DGS) (Gorges, 1986:55). En esencia, ella se originó por las controversias que surgieron en la Asociación de Política Social a propósito de las tensas relaciones entre una orientación práctica y reformista-normativa ("moralista"), por un lado, y una cientificidad metódicamente rigurosa, por el otro, entre los cuales Max Weber se destacó, sobre todo, como el defensor de una objetividad científica "libre de juicios de valor" (Wertfreiheit). Esencialmente, la fundación fue llevada a cabo por Ferdinand Tönnies, Max Weber, Georg Simmel, Werner Sombart, Franz Oppenheimer y Alfred Vierkandt, todos -exceptuando Ferdinand Tönnies- pertenecían a esta altura directamente a la Universidad Federico Guillermo (Simmel, Vierkandt, Oppenheimer) o bien habían obtenido en esta su habilitación (Sombart, los hermanos Weber).

La gran influencia que tuvo la Universidad antes de la Primera Guerra Mundial sobre la institucionalización y consolidación disciplinaria de la investigación en las ciencias sociales se hace visible por el específico rol que jugaron la Asociación de Política Social y la Sociedad Alemana de Sociología (DGS) con los científicos vinculados a la Universidad de Berlín. Aunque, la Universidad se resistió con vehemencia a la institucionalización de la disciplina. Una respectiva solicitud de la DGS por establecer una cátedra de sociología fue desaprobada con referencia a la cátedra de filosofía de Ernst Troeltsch (AUUH, 1914:254)

En esencia, fueron cuatro los científicos que se dedicaron a la enseñanza de temas sociológicos en la Facultad de Filosofía de la Universidad Federico Guillermo durante el período previo a la Primera Guerra Mundial:

- Georg Simmel hasta 1914 (se marchó a la Universidad de Estrasburgo),

- Alfred Vierkandt, en 1900 Privatdozent, desde 1913 profesor extraordinario,

- Franz Oppenheimer, desde 1909 y hasta su marcha en 1919 hacia Frankfurt,

- Kurt Breysig (1866-1940) (Stölting, 1986:111; Käsler, 1984:409), desde 1892 Privatdozent y, desde 1896, profesor extraordinario.

Durante esta fase, Simmel fue la figura más visible tanto nacional como internacionalmente. Su aproximación a la investigación se caracterizó por una perspectiva 
"analítica”, según la cual, la sociología descomponía inicialmente determinados objetos de la indagación, y luego "solo tras su propio concepto (esto es, de la sociología, H.W.) lo recompone en uno" (Nedelmann, op. cit. 131). Entre sus obras centrales figuran, la Filosofía del dinero (1900), al igual que Las grandes ciudades y la vida espiritual (1903), con lo cual se convirtió en el fundador de la sociología urbana. Sin embargo, no legó ningún sistema filosófico o sociológico consistente, ni tampoco una escuela. No obstante, fue nombrado junto a Max Weber y Ferdinand Tönnies como uno de los tres sociólogos más influyentes (makers) en las entrevistas realizadas en 1934 a ocho de los sociólogos alemanes más conocidos por el científico social americano, Earle Edward Eubank (Eubank, 1934:36)

\section{EL DESARROLLO DE LA SOCIOLOGÍA ENTRE 1918 Y 1933}

Tras la revolución de noviembre de 1918, los prusianos "republicanos" se posicionaron, de aquí en más, como los dispositivos políticos de una nueva política científico-universitaria. A partir de 1919, la política científico-universitaria fue diseñada decisivamente por Carl Heinrich Becker (1876-1933). Al inicio, en 1916, profesor en estudios orientales y del medio Este en Berlín y referente en el Ministerio Prusiano de Cultura, tras la revolución de noviembre de 1918 fue llamado por el Ministro de Cultura, Konrad Haenisch (SPD), Subsecretario de Estado en abril de 1919, de 1925 a 1930 fue Ministro Prusiano de Cultura bajo el Primer Ministro Otto Braun (SPD). Luego de su "pensamiento para la reforma de la educación superior" (1919), la democratización del estatuto universitario fue "núcleo central de toda reforma académica", en particular por medio de la equiparación de los Privatdozenten con los ordinarios. Además, intentó superar la especialización disciplinaria de la "ciencia sintética", caracterizada así por él, por medio del fortalecimiento de la sociología y la ciencia política.

La nueva política de educación superior se condensó en una significativa expansión de las posiciones docentes en sociología. Esto se visualizó con énfasis también en la Universidad Federico Guillermo.

Sin embargo, el potencial docente sociológicamente relevante sufrió de sentidas pérdidas con la marcha de Simmel a la Universidad de Estrasburgo en 1914 y con el nombramiento de Oppenheimer a una cátedra a la universidad de Frankfurt a. M. en 1919. Esta fue la primera cátedra en Alemania que introdujo en su denominación "sociología". (Uno de los doctorandos más conocidos de Oppenheimer fue Ludwig Erhard, posterior Ministro Federal de Economía y Canciller Federal. Por instigación de Erhard, Oppenheimer fue destacado, como único sociólogo alemán, con una estampilla especial en la serie "Alemanes importantes").

No obstante, se sustituyó con una considerable expansión de los cargos por medio de una "revalorización" profesoral de los cargos preexistentes y mediante la creación de nuevos:

- Sombart, aún en el Instituto Superior de Comercio de Berlín, fue nombrado como profesor extraordinario en 1918 y nuevamente como profesor ordinario en 1919 (en sucesión de Adolf Wagner).

- Alfred Vierkandt, desde 1913 como Privatdozent, fue nombrado inicialmente en 1921

Profesor extraordinario de filosofía, sociología y etnología (Völkerkunde) y en 1925, profesor ordinario de sociología. (Por lo tanto, emergió por primera vez en Berlín la "sociología" como designación exclusiva de cátedra). 
- Heinrich Cunow (1862-1936), periodista de "izquierda" y político del Partido Socialdemócrata de Alemania (SPD), fue nombrado nuevamente en 1919 profesor extraordinario de estudios de los pueblos (Völkerkunde).

- Kurt Breysig, profesor extraordinario desde 1896, se convirtió en 1923 en profesor ordinario de historia universal y doctrina societal.

- El etnólogo, Richard Turnwald fue nombrado en 1926 profesor extraordinario de etnología, psicología de los pueblos y sociología.

Si bien, hasta 1919 la Facultad de Filosofía no dispuso de ninguna cátedra ordinaria de sociología, a partir de ese momento existieron tres, de las cuales Sombart tuvo la primera denominada de "sociología". En adelante, el número total de cátedras se elevó a cinco. El incremento de cargos por medio de la pluma de Becker indicó sobre todo el apoyo personal a Cunow y a Breysig.

También, en los demás institutos superiores berlineses de la región creció el número de cargos próximos a la sociología: en 1926, Götz Briefs fue llamado para la cátedra de teoría económica política y sociología industrial en el Instituto Superior Técnico de BerlínCharlottenburgo, en donde fundó en 1928, junto a Paul Riebensam, el Instituto de Sociología Industrial y de Teoría Social de la Industria (Wagner, 1990:342), en 1934 emigró hacia los EE.UU. Durante los años veinte, también vino Karl Dunkmann (Stölting, 1986; 114; König, 1987:283; Wagner, 1990:341) al Instituto Superior Técnico, en donde fundó un Instituto de Sociología aplicada. En el Instituto Superior de Comercio, desde 1926 instituto superior científico constituido por un rectorado y con el derecho a conferir el grado de doctor, estuvieron en actividad Franz Eulenburg, profesor ordinario de sociología desde 1932, y Konrad Mellerowicz, sociólogo y economista (Zschaler, 1996)

En tanto que, se puede comprobar con absoluto éxito el proyecto de reforma de Becker por promocionar la sociología en los instituto superiores (no ya como "ciencia sintética" sino como disciplina singular), su intención por fundar también la ciencia política en los institutos superiores naufragó, -probablemente por sus implicaciones filo-republicanas y democráticascon el repudio mayoritario de los conservadores y escépticos republicanos, cuando no con el de un profesorado hostil. De esta forma, se esforzó por concretar su concepto de reforma pedagógica de las "escuelas estatales burguesas" por fuera de las estructuras tradicionales universitarias

En 1920, enraizado "dentro del entorno político socialdemócrata-republicano" (Wagner, 1990:339) y promovido, considerablemente, por el compromiso personal de Becker, vio la luz el Instituto Superior Alemán de Política (Deutsche Hochschule für Politik, DHfP) (para el desarrollo de la DHfP ver Lehnert 1991: 65-93 y Bleek 2001: 198-228). A ella pertenecieron junto a Becker mismo, entre otros, Hugo Preuss, Max Weber, Friedrich Naumann, Otto Heinrich von der Gablentz. La idea originaria fundamental era la de crear un establecimiento que contribuyese al mejoramiento de la educación general de los ciudadanos y que, simultáneamente, elevase la formación política de los trabajadores del partido como las calificaciones político-administrativas de los funcionarios públicos (Wagner, 1990:339; Wagner y Wollman, 1991:65).

El interés en el análisis científico-político no estuvo inicialmente en un primer plano, no obstante, en el transcurso de los años veinte se fue haciendo cada vez más acentuado hasta que finalmente se establecieron unos requisitos de admisión y un examen de diplomatura y se instaló en 1932 un departamento de investigación. Para empezar, los cursos fueron ofrecidos voluntariamente por científicos y practicantes, quienes estaban activos a tiempo completo en otros lados (en la Universidad Federico Guillermo, en el Instituto Superior de Comercio, en 
la Administración, etc.); entre ellos estaba el experto constitucionalista, Hermann Heller (1891-1933, de origen judío, profesor extraordinario de derecho constitucional en Berlín, 1928-1932, profesor en la Universidad de Frankfurt, exiliado en 1933), el político liberal y periodista, Theodor Heuß (1884-1963, director de estudios y docente en la DHfP, 19201933, también diputado parlamentario del partido liberal-democrático alemán, 1930-1933 y de 1949 a 1959 primer Presidente de la República Federal), el periodista y teórico social, Erich Niekisch (1889-1967), diputado por el Partido independiente socialdemócrata de Alemania en el Parlamento de Bavaria, 1919-1922, periodista de "izquierda", en prisión por resistencia contra el régimen nacionalsocialista, 1937-1945, luego de 1945 profesor en la Universidad de Humboldt, 1948-1963), los historiadores Hans Delbrück, Hajo Holborn, Echkart Kehr, el jurista y funcionario ministerial Arnold Brecht (1884-1977, funcionario ministerial prusiano, 1918-1933, tras emigración en 1933 profesor de ciencia política en EE.UU., en 1945 co-fundador de ciencia política en la República Federal) y el politólogo, Sigmund Neumann (1904-1962, docente en el DHfP, 1920-1929, tras emigración en 1933 profesor de ciencia política en los EE.UU., a partir de 1948 co-fundador de ciencia política en la República Federal, docente en el Instituto Otto-Suhr de la Universidad Libre de Berlín).

Como parte del movimiento por una educación superior popular (Volkshochschule), que también surgió del compromiso sindical-socialdemócrata por la educación de adultos y trabajadores, y que hizo pie progresivamente en otras ciudades (p. ej. en Viena), se estableció en 1920 el Instituto Superior Popular del Gran-Berlín, cuyo director, Theodor Geiger (18911952), desempeñó durante ochos años un trabajo de organización y sentó, simultáneamente, las bases para una carrera de sociólogos autodidactas (Geißler y Meyer, 1999:278-295). "Como partidario de izquierda convencido de la democracia de Weimar, perteneció a aquellos casos excepcionales entre los profesores predominantemente conservadores de una "República sin republicanos" (op. cit. 279). Geiger fue nombrado en 1928 para una cátedra ordinaria de "Sociología" en el Instituto Superior Técnico de Braunschweig y emigró hacia Dinamarca en 1933.

De este modo, se desplegó durante los años veinte en Berlín un paisaje científico e investigador en ciencias sociales de una amplitud y variedad considerable en términos institucionales y de personal. De las cuarenta cátedras que fueron reportadas (Wagner, 1990: 228) en los institutos superiores alemanes luego de 1919 -por una "revaloración" de los cargos preexistentes o la creación de nuevos-, las que por regla general la denominación "sociología" estuvo vinculada con otra disciplina, seis corresponden a Berlín, y de estas nuevamente cuatro a la Universidad Federico Guillermo (Käsler, 1984:626). Por ello, Berlín ocupó en su totalidad al igual que la Universidad una posición puntera entre las ciudades alemanas (op. cit. 229).

Las entrevistas realizadas (ya mencionadas arriba) por el científico social, Earle Edward Eubank, en 1934 a los, según él, 24 sociólogos europeos más importantes y valorados (Eubank, 1934:36), suministran una mirada reveladora. Entre estos 24 sociólogos europeos, 11 eran alemanes, de los cuales dos fueron clasificados dentro del contexto berlinés (Sombart, Vierkandt). De acuerdo, al "ranking" que Eubank montó a partir de la pregunta por los sociólogos (makers) más influyentes, las mayores menciones recayeron, tal como arriba ya se mencionó, sobre Ferdinand Tönnies (junto a los fallecidos, Georg Simmel en 1918 y Max Weber en 1920).

Un testimonio impresionante sobre la productividad colectiva de los sociólogos agrupados a principios de la década de 1930 en Alemania fue el Pequeño Diccionario de Sociología elaborado por Alfred Vierkandt en los tardíos años veinte y editado por él en 1931 con un total de 62 entradas de autores renombrados (König, 1987:259), de los cuales un tercio 
provenían de la escena científica berlinesa, entre cuyos artículos seis eran de Sombart y cinco de Vierkandt y Theodor Geiger cada uno. El Pequeño Diccionario de Sociología tenía como meta -según se dice en la "Introducción"-, “definir la posición actual del movimiento científico (de la Sociología) en Alemania, en cierto modo, a través del acto de la codificación" (op. cit. 261). Incluso, cuando la edición del Pequeño Diccionario vierkandtiano fue un emprendimiento en el que estuvieron implicados sociólogos de diversas instituciones berlinesas (tanto Theodor Geiger, Götz Briefs), la cooperación científica al interior del terreno de la investigación berlinesa difícilmente pudo ir más allá de ciertas aproximaciones. Tampoco pudo emerger en la Universidad, prescindiendo del Seminario científico-estatal de orientación económica establecido en 1886, un Instituto enfocado en lo científico social (Stölting, 1986:117).

La falta de un instituto de investigación en ciencias sociales con peso en Berlín, sobre todo en la Universidad, contrastó notoriamente con el desarrollo de las Universidades de Frankfurt am Main y Colonia, en las que no se trató directamente de establecimientos estatales. Por un lado, se creó en 1924 -a partir de una donación de fondos privados- el Instituto de Investigación Social en Frankfurt, desde el cual se desarrolló -inicialmente bajo la dirección de Carl Grünberg y desde 1930 de Max Horkheimer- la Escuela de Frankfurt. La Universidad de Colonia, de gestión pública municipal (que había sido cerrada durante la ocupación napoleónica en 1810, sin embargo, después de que Renania fuese anexada a Prusia en 1815 no fue reabierta por la Administración Científica Prusiana) fue fundada nuevamente en 1919. Bajo una vigorosa cooperación del entonces primer alcalde, Konrad Adenauer se estableció el Instituto de Investigación Social. Leopold von Wiese, quien había obtenido su habilitación en 1905 en Berlín, fue nombrado como director del departamento de Sociología en este instituto y, simultáneamente, designado profesor ordinario "de Ciencia Económica Estatal y Sociología” (Alemann, 1976:649-673; Gorges, 1986:97).

Aunque, la "doctrina de las relaciones" formulada por Leopold von Wiese pareció estar en un primer plano como main stream, sobre todo en las Conferencias de las Jornadas de los Sociólogos Alemanes (y así en parte también fue percibido en el exterior) (Eubank, 1934: 159) a principios de los años treinta, más bien se distinguió por una pluralidad de enfoques y corrientes. En palabras de Werner Sombart (en retrospectiva a 1934), no había "ninguna 'sociología', sino muchos 'sociólogos'. Aquí, la sociología no se enseña en institutos o facultades específicas como en América, sino por hombres que fueron algo distinto anteriormente: historiadores, filósofos, economistas nacionales, politólogos, etnólogos, etc. Rara vez, estudia uno una teoría 'pura' exclusivamente, sino siempre en conexión con sus áreas especiales de interés”. (Sombart en Eubank, 1934:98). Y, en palabras de Leopoldo von Wiese (también en retrospectiva a 1934): "En América se hace más fuertemente hincapié en la observación, la exploración empírica de proyectos concretos y datos empíricos. Por el contrario, en Alemania el interés se halla más sobre las grandes generalizaciones del mundo como totalidad" (von Wiese en Eubank, 1934:163).

\section{LA SOCIOLOGÍA BAJO LA DOMINACIÓN NAZI}

La cuestión sobre cómo afectó la toma de poder nacionalsocialista del 30 de enero de 1933 y la edificación del dominio de la violencia nazi a la posición y desarrollo de la sociología en Alemania se convirtió en los tardíos años cincuenta en objeto de vigorosas controversias. René König defendió el punto de vista, como filósofo hasta 1934 en Berlín 
luego emigró a Zurich, de que la sociología alemana había sido llevada "a una situación de inactividad total alrededor de 1933"- justo en medio de un momento en el que se encontraba ante el "inicio de una fisura de su propia represa", la que "la hubiese impulsado a nivel mundial" (König, 1958:14). Helmut Schelsky, alumno de Hans Freyer formado en Leipzig luego de 1933, se le opuso con el decisivo argumento de que antes de 1933 "la temática de nuestra sociología (había llegado) a su fin; las melodías fueron tocadas (...) y la ciencia casi no tuvo en sí misma ninguna fuerza de desarrollo" (Schelsky, 1959:36).

Por un lado, no puede caber ninguna duda de que la sociología, tal como se desarrolló en Alemania hasta el inicio de los años treinta, fue decapitada en términos intelectuales y de personal con el acceso del régimen nazi al poder tras 1933, en el que un tercio de los sociólogos que estaban activos en los institutos superiores alemanes hasta 1933 se fueron al exilio y, un tercio adicional, fue forzado al retiro y silenciado por medio de la "emigración interna", cuando no asesinados (como Franz Eulenburg y Alfred Weber) (Rammstedt, 1986).

Por otro lado, tampoco existen dudas de que la investigación sociológica de ningún modo llegó a su fin bajo el régimen nazi, sino que fue continuada e incluso expandida en términos institucionales y de personal (para el desarrollo de esta discusión cfr. Maus, 1959:72-99; Rammstedt, 1986; Wagner, 1990:343; Wagner y Wollmann, 1991:66; Klingemann, 1996). Entre 1933 hasta 1945, fueron designados como mínimo 27 profesores de sociología (Rammstedt, 1986:131) (Sobre la expansión del personal, cfr. también la nómina de los "Soziologen in Deutschland 1940/41", (Rammstedt, 1986:99 y ss). La lista está articulada en tres grupos, A, B y C, de los cuales el grupo A de científicos se mantuvo tras 1933 (predominantemente leales al régimen nazi) en los institutos superiores (en Berlín Sombart y Thurnwald) y el grupo $\mathrm{C}$ incluye a los científicos que sirvieron activamente al régimen nazi (aquí Carl Brinkmann, Ernst Wilhelm Eschmann, Fritz Lenz y Karl-Heinz Pfeffer).

Para el análisis sobre la continuidad y el posterior despliegue de la Sociología, se pueden distinguir entre 1933 y 1945 varias fases y cohortes (Rammstedt, 1986:70; Klingemann, 1985), en especial:

1) La de los simpatizantes y defensores (pro-nacionalsocialistas) de una "sociología alemana"

2) Los defensores de una "sociología como ciencia aplicada", y

3) Los defensores de una sociología "como arma" de la política expansionista y exterminadora del régimen nazi.

Estas fases son posibles de acentuarse, tanto más agudamente en el terreno de la investigación científica de Berlín y de la Universidad, en cuanto Berlín se constituyó como el centro políticamente operativo del sistema de dominación y del aparato de represión y exterminio nazi, el cual recurrió a su vez a la Universidad y a su personal a modo de recursos activos y legitimadores.

"El desenlace brutal" de la sociología lo evidencia el desarrollo en Berlín. Aquí, la sociología, en términos intelectuales y de personal, fue drásticamente diezmada desde el ascenso nazi al poder.

- En 1933, le prohibieron a Alfred Vierkandt la enseñanza. Para una mirada sobre la difícil situación vital, psicológica y financiera tras 1933, ver la entrevista a Vierkandt en Eubank (1934:68).

- En 1933, le quitaron a Heinrich Cunow su cátedra y pensión. Sus libros, al igual que los de Georg Simmel y los de muchos otros, fueron quemados el 10 de mayo de 1933 -al alcance de la vista de un gran número de estudiantes y profesores - en la plaza frente a la Universidad en Berlín. En 1936, Cunow falleció en medio de una gran pobreza.

- En 1933, Kurt Breysig se retiró prematuramente. 
- En 1933, Franz Eulenburg, docente de ciencias económicas en el Instituto Superior de Comercio y sociólogo de descendencia judía, fue detenido y en 1936 asesinado en la cárcel de la Gestapo en Berlín Alexanderplatz (König, 1987:345).

- En 1934, Götz Briefs emigró a los EE.UU.

Un gran número de docentes (con preponderancia política socialdemócrata, en todo caso, democráticos "republicanos") se fueron del Instituto Superior Alemán de Política tras su cooptación por el régimen nazi al exilio (su mayoría a EE.UU.) (Hajo Holborn, Arnold Brecht, Sigmund Neumann) o una "emigración interna" (Theodor Heuß).

Las expulsiones y represiones a los sociólogos en otros lugares de Alemania fueron dramáticamente similares. Para recordar solo algunos ejemplos. Al exilio fueron empujados: Karl Mannheim, Theodor Geiger, Max Horkheimer, a la "emigración interna": Alfred Weber, Franz Oppenheimer, Ferdinand Tönnies. Por consiguiente, la tesis a probar, ¿estuvo la Sociología cerca "de una ruptura"? En tanto, prueba a la tesis de René König puede ser visto el hecho de que, la investigación social alemana fue decapitada justo en un momento en que las mejores y más creativas cabezas de la sociología alemana fueron expulsadas al exilio a finales de la década de los veinte (y lo mismo vale para los antiguos miembros del Instituto Superior Alemán de Política (Deutsche Hochschule für Politik) y puesto que la investigación social se encontraba frente una "ruptura", estos sobresalieron por una multiplicidad de excelentes investigaciones internacionales desde el exilio.

La tesis opuesta dice: la Sociología se corrompió en tanto adaptación "pronacionalsocialista", como "sociología (popular) alemana". Por un lado, se identifica al grupo de sociólogos que mantuvieron sus cargos en los institutos superiores después de 1933, quienes se habían inclinado durante los años veinte, cada vez con más fuerza, hacia una "revolución conservadora" cuyo eje giró en torno al "pueblo y la comunidad", y quienes saludaron la llegada de los nacionalsocialistas al poder como oportunidad para establecer (y distanciarse así de la sociología francesa y anglosajona) una sociología alemana y "popular".

Dentro de esta cohorte puede ser imputado Werner Sombart (aunque con limitaciones) en Berlín, quien, a pesar de su efectivo retiro en 1931, continuó en 1933 (con 70 años) su actividad docente en la Universidad (hasta 1938). Durante los años veinte, fue renunciando progresivamente a sus antiguos cargos y girando hacia concepciones "populares" socialconservadoras. En su libro publicado en 1934 bajo el título de Deutscher Sozialismus en el que él formuló una verdadera revocación de su Sozialismus und soziale Bewegungen de 1897, Sombart mismo proclamó el "Führerstaat" entre otros, como solución a los problemas sociales. Después, sin embargo, tomó distancia del régimen nazi (ver Eubank 1934:101), sobre todo con el agudo rechazo a la teoría racista nazi (Sombart, 1984:44). Finalmente, él cayó obviamente en desgracia política y murió en 1938. Con este trasfondo la expresión de René König de que Sombart "empezó primero como marxista y terminó como nacionalsocialista" (König, 1987:26), es sin duda excesiva.

También, Richard Thurnwald, quien continuó con su actividad docente en la Universidad Federico Guillermo tras 1933, intentó llegar aparentemente a un acuerdo con el régimen nazi (cfr. el comentario amargo de René König, cuyo director de tesis de doctorado era Thurnwald: "Es muy doloroso tener que decir adiós a un profesor venerado de esta forma bochornosa", König, 1987:278).

Dentro de la cohorte ("pro-nacionalsocialista") que al menos abarcó a un tercio de los sociólogos en actividad antes de 1933, Hans Freyer, docente en Leipzig, jugó un rol clave, (ver Vierkandt, 1934) "El hombre destacado del nuevo rumbo de la sociología alemana bajo influencia del nuevo régimen es Hans Freyer (...) La nueva sociología está interesada 
únicamente en co-crear la vida política del Nuevo Estado", (Eubank 1934:71; ver también Sombart, 1934). Algunos sociólogos han llegado incluso a apoyar al nuevo status (esto es, el régimen nazi) como por ejemplo, Hans Freyer (...) En la actualidad solo aquellos de las ciencias sociales apoyan al partido (esto es, el NSDAP)" (Eubank 1934:101).

Sus alumnos y asistentes, Helmut Schelsky y Arnold Huelen, fueron activos partidarios nazis.

Otro grupo es el de "la sociología como investigación aplicada" (Rammstedt, 1986:107). Ella se juntó, durante los años veinte, con la crítica formulada a la sociología universitaria por la distancia de la práctica y aplicación, y después de 1933 se fortaleció como consecuencia de haber señalado el creciente déficit de información y conocimiento dentro del fragmentado aparato de dominación nazi, con lo que la investigación sociológica se hizo obligatoria. Esto valió sobre todo para las encuestas "internas" que aseguraban la dominación. Una "modernización intra-científica" (Wagner, 1990:343; Wagner y Wollman, 1991:66) de la sociología tuvo lugar en Alemania con la aplicación de los nuevos métodos (orientados por el estado de la investigación norteamericana) de las ciencias sociales.

Por último, apareció una cohorte de sociólogos (en especial tras el estallido de la guerra de Hitler por la ocupación y exterminio) que, en tanto adeptos (fanáticos, en muchos casos) del régimen nazi, vio a la investigación sociológica, como un "arma" (Rammstedt, 1986:114 y 137 para lo sucesivo) para asegurar la tiranía e instrumentar una estrategia de ocupación y exterminio. Por eso, los roles de científico y perpetrador se entrelazaron y mezclaron progresivamente. La cercanía espacial entre el mundo científico y político en Berlín ofreció ejemplos de combinación y confusión particularmente fatales en términos funcionales, institucionales y de personal para el despliegue de una sociología de "investigación aplicada" y su transición hacia una sociología "como arma". Por sus útiles y solicitados recursos de conocimiento, actividad y legitimidad, la Universidad se convirtió en la preferida del régimen nazi.

En primer lugar, hay que señalar aquí a la Facultad Científica Extranjera, que se estableció en 1939 como una nueva Facultad y caracterizada como la "primera Facultad de Sociología dentro de un instituto superior alemán". En su creación cooperó activamente la Reichssicherheitshauptamt, (RSHA) a cargo de Heinrich Himmler. En 1940 el Instituto Superior de Política, adaptado, reestructurado totalmente su personal y renombrado como, Instituto Superior Alemán de Política fue integrado a la nueva facultad.

Fue nombrado como decano el treintañero, Franz Alfred Six, quien había realizado una carrera meteórica en las SS., (nacido en 1909, jefe de personal en la SS, desde 1936 director de la división central de la Reichssicherheitshauptamt (RSHA) -responsable para la "observación ideológica y el combate contra el judaísmo enemigo"-, en 1939 jefe de la RSHA dirigida por Himmler, en 1943 decano de la Facultad Científica Extranjera y profesor ordinario de Política Exterior y Estudios Extranjeros, manteniendo su posición en la RSHA; ver Rammstedt, 1986:137).

Otro dirigente nacionalsocialista fue el geopolítico, Karl Heinz Pfeffer. (Nacido en 1906, docente en 1934 de Sociología en la Universidad de Leipzig, en 1940 profesor extraordinario de sociología, estudios populares y regionales de Gran Bretaña, Facultad Científica Extranjera, en 1942 profesor ordinario de estudios populares y regionales de Gran Bretaña y del Imperio Mundial, en 1943 decano y presidente representativo del Instituto Científico Extranjero de Berlín -.como sucesor de Six) de la Facultad Científica Extranjera. En el pensamiento de Pfeffer, la Sociología solo "(será) real como expresión necesaria del ser popular, cuando (sea) el arma de un pueblo combatiente.” (Rammstedt, 1986:116). 
Con el estallido de la guerra, la ocupación territorial de Europa del Este por la Alemania de Hitler generó áreas de trabajo fundamentales para la Facultad, subordinadas directamente al jefe (Reichsfiuhrer) de la SS Heinrich Himmler. Otros profesores de la Facultad Científica Extranjera fueron el sociólogo, Carl Brinkmann (1885-1954, profesor ordinario en 1923 de Economía y Sociología en la Universidad de Heidelberg, desde 1942 profesor ordinario en Berlín (cfr. Leopold von Wiese) "Carl Brinkmann en Heidelberg es "hombre de partido" (es decir, del NSDAP). "Él se ha apartado totalmente de su postura democrática anterior", en Eubank (1934:162) y Ernst Wilhelm Eschmann (nacido en 1904, docente en el Instituto Superior Alemán de Política en 1933, periodista en Berlín - Tat-Kreis, catedrático suplente en la Facultad Científica Extranjera, en 1943 profesor extraordinario de Estudios Populares y Regionales de Francia).

Además, hay que referirse al Instituto de Investigación Estatal. Su director fue Reinhard Höhn de 31 años en 1935, quien además había sido nombrado en 1939 profesor ordinario de derecho público en la Facultad Jurídica. Simultáneamente desde 1934, Höhn fue colaborador exclusivo del servicio de seguridad estatal y se mantuvo allí como dirigente en la Reichssicherheitshauptamt (RSHA) bajo un rango jerárquico de la SS hasta el fin de la guerra. En el servicio de seguridad, Höhn organizó la denominada cobertura de áreas vitales, la que representó una primera forma institucionalizada del "sistema de alerta temprana" apoyada sobre sucesivos cuestionarios de base científico-social (Klingemann, 1996:30).

Especialmente, la Universidad Federico Guillermo, mediante la Facultad Científica Extranjera y el Instituto de Investigación Estatal, sus profesores y colaboradores, se involucró dentro del aparato represivo y exterminador nazi de una forma siniestra y criminal, y lo hizo sujeta a su servicio. Al término de esta traición a la responsabilidad e integridad científica sin precedentes por una amplia parte de la Universidad de Berlín estuvo el exterminio material, humano y moral del que quedó poco más que ruinas en mayo de 1945.

\section{POSTSCRIPTUM}

A pesar de estar involucrados en el régimen nazi y en sus operaciones criminales, no pocos de estos malhechores continuaban en la posguerra una carrera a veces espléndida. Por ejemplo Reinhard Höhn se convirtió en 1956 en director de la Academia Bad-Harzburg para el Personal Ejecutivo de la Economía, una influyente escuela de gerencia en la República Federal. Con motivo de su 95 cumpleaños, Reinhard Höhn fue elogiado, en 1999, por el presidente de la Asociación Federal de las organizaciones patronales alemanas en un acto festivo.

\section{BIBLIOGRAFÍA}

ALEMANN, H. von (1976). "Leopold von Wiese und das Forschungsinstitut für Sozialwissenschaften in Köln 1919-1934” en Kölner Zeitschrift für Soziologie und Sozialpsychologie, 28: 649-673.

AUUH, Archivo universitario de la Universidad de Humboldt, $\mathrm{n}^{\circ}$ 34, protocolo del 25. 06. 1914, tomo 1. BAUMGARTEN, M. (1997). Professoren und Universitäten im 19. Jahrhundert. Zur Sozialgeschichte deutscher Geistes- und Naturwissenschaftler. Göttingen: Vandenhoeck und Ruprecht.

BEIN, A. (s.d.). Franz Oppenheimer als Mensch in Zionist, disponible en: http://franz-oppenheimer.de/ xbein1.htm. 
BICKEL, C. (1999). "Ferdinand Tönnies". En D. Käsler (ed.) Klassiker der Soziologie vol. 1, Vom August Comte bis Norbert Elias München: Verlag C.H. Beck.

BLEEK, W. (2001). Politikwissenschaft in Deutschland. München: Verlag C.H. Beck.

BRUCH, R. (1980). Wissenschaft, Politik und öffentliche Meinung. Gelehrtenpolitik im Wilhelminischen Deutschland (1890-1914). Husum: Matthiesen Verlag.

FAATZ, A. (2002). "Hugo Preuß”. En M. Fröhlich (ed.) Die Weimarer Republik: Portrait einer Epoche in Biographien. Darmstadt: Primus-Verlag, pp. 15-27.

GEIßLER, R. y MEYER, T. (1999). “Theodor Geiger”. En D. Käsler (ed.) Klassiker der Soziologie vol. 1, Vom August Comte bis Norbert Elias. München: Verlag C.H. Beck.

GERHARDT, V.; MEHRING, R. y RINDERT, J. (2000). Berliner Geist. Eine Geschichte der Berliner Universitätsphilosophie. Berlin: Akademie-Verlag.

GORGES, I. (1986). Sozialforschung in Deutschland 1872-1914. Meisenheim: Anton Hahn Verlag.

KÄSLER, D. (1984). Die frühe deutsche Soziologie 1909 bis 1934 und ihre Entstehungs-Milieus. Opladen: Westdeutscher Verlag.

KÄSLER, D. (1985). Soziologische Abenteuer. Earle Edward Eubank besucht europäische Soziologen im Sommer 1934. Opladen: Westdeutscher Verlag.

KLINGEMANN, C. (1996). Soziologie im Dritten Reich. Baden-Baden: Nomos-Verlags-Gesellschaft.

KÖNIG, R. (1958). "Einleitung" en Soziologie. Frankfurt a.M.: Fischer-Lexikon.

KÖNIG, R. (1987). Soziologie in Deutschland. München: Carl Hanser.

LEHNERT, D. (1991). "Schule der Demokratie" oder "politische Fachhochschule"? Anspruch und Wirklichkeit einer praxisorientierten Ausbildung der deutschen Hochschule für Politik 1920-1933". En G. Göhler y B. Zeuner (eds.) Kontinuitäten und Brüche in der deutschen Politikwissenschaft. BadenBaden: Nomos.

LENGER, F. (1994). Werner Sombart 1863-1941. Eine Biographie. München: Beck.

MAIER, H. (1980). Die ältere deutsche Staats- und Verwaltungslehre. München: C.H. Beck.

MAUS, H. (1959). "Bericht über die Soziologie in Deutschland 1933 bis 1945", en Kölner Zeitschrift für Soziologie und Sozialpsychologie, 11: 72-99.

NEDELMANN, B. (1999). "Georg Simmel”, en D. Käsler (ed.) Klassiker der Soziologie vol. 1, Vom August Comte bis Norbert Elias. München: Verlag C.H. Beck.

OPPENHEIMER, F. (1929). "Selbstdarstellung". En von F. Meiner (ed.) Die Volkswirtschaftslehre der Gegenwart in Selbstdarstellungen, vol. 2 Selbstdarstellungen. Leipzig: Verlag von Felix Meiner.

RAMMSTEDT, O. (1986). Deutsche Soziologie 1933-1943. Die Normalitat einer Anpassung. Frankfurt a. M.: Suhrkamp.

SCHELSKY, H. (1959). Ortsbestimmung der deutschen Soziologie. Düsseldorf/Köln: Eugen Diederichs Verlag.

SIGMUND, S. (1993). “Georg Simmel in Berlín” en Berliner Journal für Soziologie, 3: 161-181.

SOMBART, N. (1984). Jugend in Berlin: 1933-1943, Ein Bericht. München/Wien: Hanser.

STÖLTING, E. (1986). Die akademische Soziologie in der Weimarer Republik. Berlin: Duncker \& Humbolt.

STÖLTING, E. (1999). "Robert Michels". En D. Käsler (ed) Klassiker der Soziologie vol. 1, Vom August Comte bis Norbert Elias. München: Verlag C.H. Beck.

TÖNNIES, F. (1922). "Selbstdarstellung". En F. Meiner, Felix (ed.) Die Volkswirtschaftslehre der Gegenwart in Selbstdarstellungen vol. 2 Selbstdarstellungen. Leipzig: Verlag von Felix Meiner.

WAGNER, P. (1990). Sozialwissenschaften und Staat. Frankreich, Italien, Deutschland 1870-1980 Frankfurt a. M../New York: Campus Verlag.

WAGNER, P. y WOLLMANN, H. (1991). "Beyond Serving State and Bureaucracy: Problem-oriented social science in (West) Germany", en Knowledge and Policy 4, Cuaderno 1-2.

ZSCHALER, F. (1996). Vom Heilig-Geist-Spital zur Wirtschaftswissenschaftlichen Fakultät: 110 Jahre Staatswissenschaftlich-Statistisches Seminar an der vormals Königlichen Friedrich-Wilhelms Universität, 90 Jahre Handels-Hochschule Berlin. Berlín/Heidelberg: Springer Verlag.

ZSCHALER, F. (1996/97). "Wo Kaufleute ihr Handwerk lernten ..." en Humboldt-Zeitung 17 Oktober, Berlin: Der Alma Mater Berolinensis. 


\section{Breve currículo:}

\section{Hellmut Wollmann}

Profesor emérito, Doctor en Derecho, abogado y politólogo. Estudió en las Universidades de Heidelberg y Berlín de Alemania y Wesleyan, Connecticut, EE.UU. Fue Kennedy Memorial Fellow en la Universidad de Harvard (1970-1971). Profesor de Ciencias de la Administración en la Universidad Libre de Berlín (1974-1993) y en la Universidad Humboldt de Berlín (1993-2001). Profesor visitante en numerosas Universidades (Beijing, Tokio, Moscú, Florencia, Gotemburgo, entre otras). Ha investigado y publicado trabajos sobre estudios comparativos de política y administración pública (con un enfoque local), investigación evaluativa y sociología de la ciencia.

*Texto traducido por:

\section{Jacqueline M. Rajmanovich}

Licenciada en Sociología por la Facultad de Ciencias Sociales de la Universidad de Buenos Aires, Argentina, investigadora tesista en el Instituto de Investigaciones Gino Germani (Sociales UBA). Finalizando sus estudios de Maestría en Gestión Educativa en la Universidad Nacional de General San Martín (UNSAM). Es docente del Profesorado en Sociología (Sociales UBA) y del Instituto Superior de Formación Docente No 39 (ISFD Nº 39). 